\title{
Anisotropic water diffusion in nematic self-assemblies of clay nano-platelets suspended in water.
}

\author{
E. N. de Azevedo, ${ }^{*}$ M. Engelsberg, ${ }^{\dagger, \ddagger}$ J. O. Fossum, ${ }^{\S}$ and R. E. de Souza ${ }^{\ddagger}$. \\ *Programa de Pós-Graduação em Ciência de Materiais, Universidade Federal de Pernambuco, \\ 50670-901, Recife, Pernambuco, Brazil. \\ ${ }^{\ddagger}$ Departamento de Física, Universidade Federal de Pernambuco, 50670-901, Recife, Pernambuco, \\ Brazil.

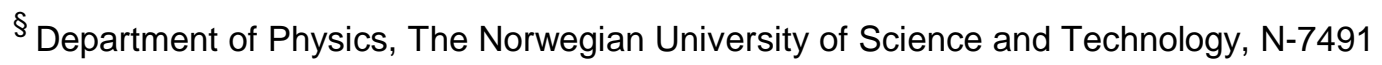 \\ Trondheim, Norway.
}
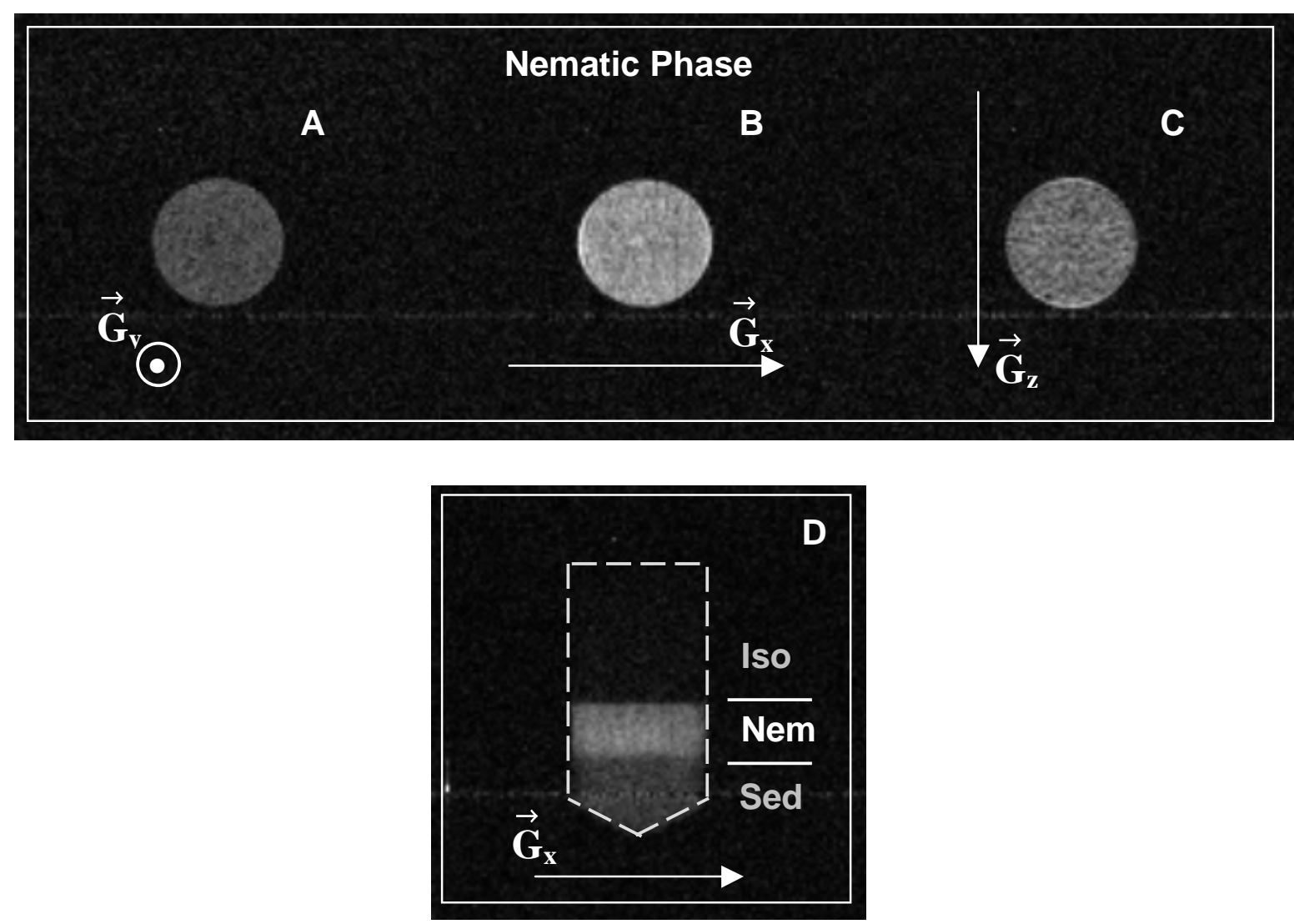

Figure S1 Transverse diffusion weighted images of the nematic phase region of a $\mathrm{Na}$-Fht colloidal supension within a cylindrical container with Teflon walls and no applied magnetic field. Magnetic field gradients along (A) y, (B) $x$, and $(C) z$ axis. Longitudinal diffusion weighted image showing the three phases for a Na-Fht suspension within a container with Teflon walls. (D) Magnetic field gradient is along the $x$ axis. 